\title{
Energy Efficient Receiver Signal Strength Indicator Based Clustered Routing Algorithm for Wireless Sensor Network
}

\author{
R. Anandha Lakshmi, T. Sureshz, R. Maruti
}

\begin{abstract}
Robust and efficient algorithms for routing and other process for a wireless sensor network are under active development due to technological advancements on wireless transmission systems. Each of the sensor nodes in a wireless sensor network either transmits or forwards the data packets to the base station. The main objective of the majority of the work in the literature is to save the energy consumption efficiently. The cluster based routing mechanism helps to achieve low energy consumption within the network. The network organizes its nodes as a cluster and selects a particular node as cluster head to manage the transmission within and between clusters. The majority of the clustering approach selects the cluster head using a thresholding based approach. Nodes having energy level higher than the threshold are the candidates for the cluster head selection. In the proposed approach the nodes remaining energy and the sum of distance between individual nodes to the cluster head node is considered. Optimal cluster head selection will help to increase the overall life time of the network. The distance between the sensor nodes is estimated using RSSI (Received Signal Strength Indicator) and other parameters measured from the physical layer. Experiments are conducted with simulation environment created with the NS-2 simulator and efficiency of the approach is analyzed in detail.
\end{abstract}

Keywords: cluster based routing, RSSI (Received Signal Strength Indicator), NS-2 simulator, cluster head selection, thresholding.

\section{I.INTRODUCTION}

A Wireless Sensor Network (WSN) comprises of a set of sensor nodes either mobile or static in nature. The sensor nodes communicate among each other through a wireless communication link. Each sensor node is equipped with low processing power, limited memory, and bandwidth. These nodes sense the environment, collect the relevant data, and then forward them to the sink or base node. The communication between the sensor node and base station is trivial in the design of routing protocols for WSN. Majority of the routing approaches follow a hierarchical cluster based routing by which the sensor nodes are grouped together to form a cluster.

Revised Manuscript Received on February 05, 2020

* Correspondence Author

Ms. R.Anandha Lakshmi, Assistant Professor, Department of BCA, Anna Adarsh College for Women, Chennai. anandhalakshmi.sk@gmail.com

Dr. T. Suresh, Associate Professor, Department of Computer Science and Engineering, Annamalai University, Chidambaram, Tamil Nadu. sureshaucse@gmail.com

Dr. R. Maruti, Professor, PonnaiyahRamajayam Institute of Science and Technology, Chennai. rmaruthi2014@gmail.com

(c) The Authors. Published by Blue Eyes Intelligence Engineering and Sciences Publication (BEIESP). This is an open access article under the CC BY-NC-ND license (http://creativecommons.org/licenses/by-nc-nd/4.0/)
The communication between the sensor node and base station is accomplished through a multi-hop routing approach by forwarding the data packets through the intermediate cluster heads. The design of the WSN routing protocols and other algorithms poses several challenges due to its resource constrained environment. One of the biggest challenges of WSN is the efficient utilization of energy available in the sensor nodes. The data transmission from the sensor node to the base station does consume more energy if each node communicates with the base station through a single hop communication. Energy efficient communication strategy is to follow geographic location based routing which greatly differs from the hierarchical routing [1]. By this approach the location of each sensor node is modeled using mathematical model. The existing node location estimation schemes uses GPS devices to find the sensor nodes location but the approach is not energy efficient [2]. Some of the literatures have adopted RSSI based wireless sensor node localization schemes which are considered to be energy efficient when compared to the methods which uses modern sophisticated devices. When the mobile nodes are static in nature then the node localization scheme does not consume more power but when the nodes are mobile in nature then they consume more energy as the node location has to be tracked continuously. Experimentally the RSSI is observed to be inconsistent even in a constrained and controlled environment [3]. But the LQI can effectively describe the error rate and when RSSI is combined with LQI then the distance estimation will be more accurate [4]. This paper attempts to utilize the combination of RSSI and LQI techniques to measure the distance between the nodes and the hypothesis is verified experimentally through simulations.

\section{II.RELATED WORKS}

This section presents the review and analysis of various clustering approaches adopted in literature using RSSI. In [6] an improved node localization scheme is proposed which maintains low energy consumption in clustering phase is proposed. In [7] a cluster head selection mechanism is performed by calculating the distance between the sensor nodes joined in the cluster. The distance is estimated using the RSSI metric. The RSSI of each node to the sink node is used for cluster formation in wireless sensor network [8]. The problems prevailing in LEACH routing protocol including packet forwarding and cluster head node selection is resolved. 
A distance measurement algorithm based on hybrid filter and least squares dynamic estimation of environmental parameters is proposed for RSSI-based wireless sensor network localization in logarithmic normal distribution model [9]. In [10] with anchor node as reference node,the RSSI value is optimized by applying hybrid filter based on mean filter,median filter and Gaussian filter.

\section{III.PROBLEM STATEMENT}

Let us consider a Wireless Sensor Network arranged in a two-dimensional area which is denoted as a graph $G(V, E)$. The set of sensor nodes are represented $\mathrm{V}=\{\mathrm{V} 1, \mathrm{~V} 2, \ldots$, $\mathrm{Vn}\}$. The transmission range is represented as $\mathrm{R}$ which fixes maximum distance between two communicating nodes. If the distance between the two is greater than $\mathrm{R}$ then one-hop or direct communication between them is not possible. It can also be inferred that if the distance between the two nodes are greater $\mathrm{R}$ then they are not neighboring nodes. The graph $\mathrm{G}$ denoting the network can be Euclidean in which all the nodes located within the two dimensional space has a coordinate $\left(x_{i}, y_{i}\right) \in R^{2}$. The idea is to estimate the location of the sensor nodes with respect to the position of the beacon or reference nodes using RSSI and the distance estimation mechanism.

\section{IV.DISTANCE BASED CLUSTERING MECHANISM}

The clustering approaches reviewed in the literature have many issues and challenges to be addressed. The clustering approach should be scalable in nature and should have less communication overhead. The proposed clustering mechanism using distance measure is explained in this section where in the distance between the sensor node and the sink node is estimated based on a beacon signal sent from the base station. The sensor node calculates the distance between the sink node and itself based on received signal strength and link quality. In general the range based approaches for estimation of distance relies on the time of arrival (TOA), angle of arrival (AOA), RSSI, and LQI metrics. Based on the propagation time of the transferred data packets the distance is estimated in when the TOA, $\mathrm{AOA}$, and other similar techniques are used. In the case of AOA additional antennas are used to find the direction of arrival. The proposed method adopts a combination of RSSI and LQI metrics for distance estimation for improved accuracy when compared to only RSSI based distance estimation. The RSSI is a better link quality estimator and LQI can be predicted based on a pre-trained machine learning model. The use of LQI increases the likelihood of accurate distance estimation. Initially the set of possible distance and the expected power level are identified. Then using LQI values the identified set is filtered. When the value of LQI is high then the likelihood of accurate estimation of distance is higher [5].

Next the cluster heads are selected based on the following parameters remaining energy in the node, centrality of the node, and sum of distance between the candidate node and its n-neighboring nodes. The residual or remaining energy in the node is considered as the node will be a part of inter and intra cluster communication when it becomes a cluster head. The average sum of distance between the candidate node and its n-neighbors describes its proximity to the neighboring nodes. The centrality of the nodes is considered since it may help to select $\mathrm{CH}$ with low energy consumption during aggregation and forwarding of data packets.

$$
\begin{aligned}
& \text { NodeCentrality } \\
& =\frac{\sqrt{\frac{\sum_{i \in\{1,2, \ldots, n\}} d^{2}(i, j)}{n}}}{A}
\end{aligned}
$$

Where $\mathrm{A}$ is the area of the network; $\mathrm{n}$ denotes the number of neighbors considered; and $d(i, j)$ is the distance between the candidate node and the neighboring node. Based on the above mentioned parameters the final list of $\mathrm{CHs}$ is found using a decision rule. The sensor nodes join the cluster based on the nearness to the CHs. When the distance between a sensor and two different cluster heads are same the node joins the cluster from which the advertisement message came early. The cluster heads collects the data from the sensor nodes and forwards to the sink through multi-hop approach.

In the proposed clustering approach, the cluster heads will not be changed frequently. This approach helps to reduce the energy expenditure in the network. The clustering process is initiated invoked when the distance between the sensors and their respective cluster head goes above a threshold level. In a cluster-based approach the head node shall cater only to a limited set of sensor nodes (threshold denoted as ' $\delta$ ').

This limitation is due to the fact to ensure easy access of transmission channel instead of sensor nodes waiting longer time to transmit their sensed information to their respective head node. An optimal value of the degree of each cluster head helps to achieve better throughput. If the node lies within a certain transmission range then the battery power can be effectively used as only less power is consumed when a sensorneeds to forward its data to cluster head. The cluster head spends more power as it sends or forwards data packets to the base station.

The load of a head node depends rely on the sensor nodes and volume of data sent by the sensor nodes lying within the cluster. The cluster head also relays the packets from various other clusters to the base station. Perfect load balancing within the network cannot be achieved as the sensor nodes move frequently within the network region. The Load-Balancing-Factor (LBF) helps to estimate the level of data load distribution within the network. The cluster head node's load will be expressed using the density in the cluster and the variance of the node density will represent the distribution of the load. The load factor can be taken as the reciprocal of the variance of the density.

$$
L B F=\frac{n_{c}}{\sum_{i}\left(x_{i}-\mu\right)^{2}}
$$

wherenc is the count of cluster heads, xi is the density of cluster $\mathrm{i}$, and $\mu=(\mathrm{N}-\mathrm{nc}) / \mathrm{nc}$, ( $\mathrm{N}$ being the density of nodes in the system) is the average count of neighbors of a cluster head. When the load is evenly distributed within the network then the value of LBF will be higher and it will be infinite if the load is perfectly balanced [11]. 


\section{V.EXPERIMENTS AND RESULTS}

In our experiments the network is simulated with the following parameters

\begin{tabular}{|c|c|}
\hline No. of nodes & Varied from 20 to 100 \\
\hline Network Area & $500 \times 500 \mathrm{~m} 2$ \\
\hline Radio Model & Two-ray ground - Reflection model \\
\hline Initial Energy in the nodes & $10 \mathrm{~J}$ \\
\hline
\end{tabular}

The lifetime of a WSN network greatly relies on the lifetime of the sensor nodes which have limited energy resources. The main objective is to limit the energy expenditure in the network when the nodes are grouped together as a cluster and coordinated by a cluster head node. The efficiency of this approach can be analyzed by estimating the amount of energy conserved within the network.

A node spends energy in sensing and forwarding the data packets to the $\mathrm{CH}$. The $\mathrm{CH}$ node spends energy for receiving, aggregating, and forwarding the data packets. Thus the energy spent by a node can be expressed mathematically as below

$$
\text { Espent }=\text { Esense }+ \text { ETx }
$$

The energy spend by a cluster of nodes of size $\mathrm{n}$ for sensing and transmitting $\mathrm{x}$ data packets can be expressed as

$$
E_{\text {cluster }}=(n . x . t) \cdot E_{\text {spent }}
$$

Similarly the energy spent by a cluster head (ECH) for receiving, aggregation, and forwarding of data packets can be calculated as given below

$$
\begin{aligned}
& E_{C H} \\
& =n \cdot x \cdot t \cdot E_{r x}+n \cdot x \cdot t \cdot E_{D A} \\
& \left.+\frac{1}{\alpha}(n \cdot x \cdot t) E_{t x}\right)
\end{aligned}
$$

Where Erx, EDA, and Etx are the energy spent by cluster head for receiving, aggregation, and transmission respectively. The value of $\alpha$ depends on the ration of aggregation.

The total energy ECi spent in the cluster $i$ is the sum of energy spent by the $n$ cluster nodes and the cluster head. It is expected that under such environment the protocols and other algorithms should help to achieve Eh amount of energy conservation within the network.

$$
E_{h}=\frac{E_{C i}}{n+1}
$$

During the simulation period the amount of energy harvested between a time period can be calculated as

$$
\begin{aligned}
& E_{h(t 1-t 2)} \\
& =E_{t 1}+\eta \int_{t 1}^{t 2} E_{h}(n, t) d t \\
& -\int_{t 1}^{t 2} E_{l}(n, t) d t
\end{aligned}
$$

The total energy harvested during the interval $\mathrm{t} 1$ and $\mathrm{t} 2$ is calculated based on the initial residula energy in the cluster nodes, energy conserved during the interval, and the energy leaked during the interval. The energy consumed within the network must be compensated with the energy harvested in the network. Initially during the simulation of the proposed clustering approach the nodes estimate their distance and cluster heads are identifed. Then the nodes joins the nearby
Table 1: Network Simulation parameters

cluster and starts their operation. The cluster nodes communicates with the $\mathrm{CH}$ based on TDMA schedule and the corresponding $\mathrm{CH}$ transmits their aggregated data to the sink through multi-hop route.

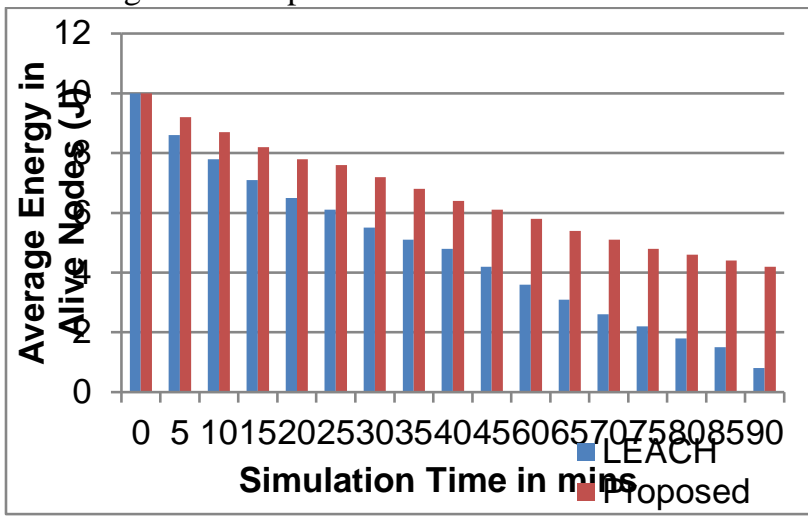

Fig.1 Average Energy in Alive Nodes

Based on the experiments and the results of the simulation the average energy remaining in the nodes during the simulation period is estimated and presented n Fig. 1. It is obvious that the average energy remaining in the nodes are increased due to distribution of energy consumption within the network uniformly. The node stability is increased and thereby the overall lifetime will be increased. The throughput of the transmissions are increased (shown in Fig. 2) as the attenuation is reduced by following short distance communication for both type of communication within the network (sensor to cluster head and $\mathrm{CH}$ to sink node).

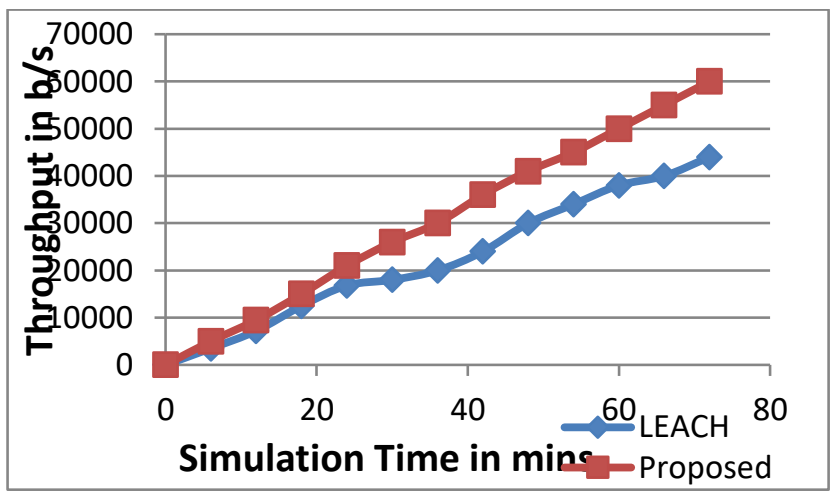

Fig.2Throughput in the Network

\section{VI.CONCLUSION}

The protocol aims at conserving energy during the transmission of sensed information to the sink node. The routing algorithm segments the network in to clusters and monitors the energy consumption in the cluster heads and the cluster nodes. The proposed method follows and efficient strategy for selecting optimal Cluster Head nodes and follows a multi-hop communication between the $\mathrm{CH}$ and the sink node. This helps to eliminate the longer distance communication in the network and hence the attenuation of the signal is limited. This reduces the number of retransmission in the network and saves more energy within the network. 


\section{Energy Efficient Receiver Signal Strength Indicator Based Clustered Routing Algorithm for Wireless Sensor Network}

The energy consumption during transmission is distributed evenly within the network and simultaneously the lifetime and the performance of the network is increased.

\section{REFERENCES}

1. Grover, Jitender, and Mohit Sharma. "Location based protocols in Wireless Sensor Network-A review." Fifth International Conference on Computing, Communications and Networking Technologies (ICCCNT). IEEE, 2014.

2. Kuriakose, Jeril, et al. "A review on localization in wireless sensor networks." Advances in signal processing and intelligent recognition systems. Springer, Cham, 2014. 599-610.

3. Guan, Bo, and Xin Li. "An rssi-based wireless sensor network localization algorithm with error checking and correction." International Journal of Online and Biomedical Engineering (iJOE) 13.12 (2017): 52-66.

4. Ermakov, A., N. Gorshkov, and A. Titaev. "Location Management of Mobile Nodes in Low-Power Wireless Sensor Network Using Link Quality Metric." CEUR Workshop Proceedings. Vol. 2298. CEURWS, 2018.

5. M. Raju, T. Oliveira and D. P. Agrawal, "A practical distance estimator through distributed RSSI/LQI processing - An experimental study," 2012 IEEE International Conference on Communications (ICC), Ottawa, ON, 2012, pp. 6575-6579.

6. El Houssaini, Dhouha, et al. "Rssi based clustering algorithm for wireless sensor network localization in large scale." Sensorica IEEE WORKSHOP. Vol. 6. 2017.

7. Karasekreter, Naim, FatihBaşçiftçi, and UğurFidan. "Rssi Based Clustering Algorithm for Wireless Sensor Networks." The Online Journal of Science and Technology-January 7.1 (2017).

8. Hong, Sung-Il, and Chi-Ho Lin. "An expansion cluster routing algorithm based on rssi for an efficient data transmission." 2016 18th International Conference on Advanced Communication Technology (ICACT). IEEE, 2016.

9. Tao, Weige, Yihua Zhu, and ZiyanJia. "A distance measurement algorithm based on RSSI hybrid filter and least square estimation." Chinese Journal of Sensors and Actuators 25.12 (2012): 1748-1753.

10. Tao, Weige, Yihua Zhu, and ZiyanJia. "A distance measurement algorithm based on RSSI hybrid filter and least square estimation." Chinese Journal of Sensors and Actuators 25.12 (2012): 1748-1753.

11. Pandey, Sudhakar, and Narendra Kumar Shukla. "Improved Weighted Clustering Algorithm for Mobile Ad Hoc Networks." International Journal of Engineering and Management Sciences 2.1 (2011): 20-25.

\section{AUTHORS PROFILE}

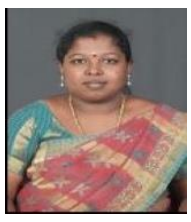

Ms. R.Anandha Lakshmi is currently working as Assistant Professor in Anna Adarsh College for women, Chennai, Tamil Nadu with 10 years of teaching experience. She is pursuing her Ph.D. Degree in Computer Science from Annamalai University. She received her M.Phil., from Mother Terasa University, Kodaikanal in the year 2008, and Degree of Master of Science from Madurai Kamaraj University, Madurai in the year 2004. She did her Bachelor's Degree in Computer Science from Madurai Kamaraj University, Madurai in the year 2002. She has published various research papers in reputed International journals.

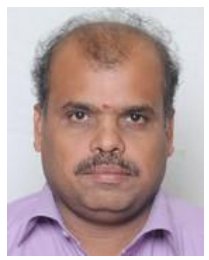

Dr. T. Suresh received his Bachelor of Engineering Degree in Computer Science and Engineering from Shanmuga College of Engineering, Thanjavur. He did his M.B.A degree from Madurai Kamaraj University. He did his post-graduation and Ph.D. Degree in Computer Science and Engineering from Department of Computer Science and Engineering, Faculty of Engineering and Technology from Annamalai University. He has contributed around 40 technical papers and in various journals \& conferences. He is currently working as an Associate Professor in the Department of Computer Science and Engineering, Annamalai University, Annamalai Nagar, Tamil Nadu, India. His area of interest includes MANET, VANET, WSN, Cloud Computing etc.

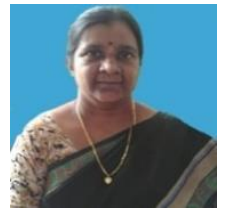

Dr. R. Maruthi received her Bachelor'sDegree and Master of Computer Applications from Bharathidasan University in the year 1997 and 2000 respectively. She completed her M. Phil from Alagappa University in the year 2008 and Ph.D. Degree in Computer Science from Mother Terasa Women University in the year 2011. She has contributed more than 15 research papers for indexed journals. She has more than 17 years of teaching experience and currently working as Professor in PRIST University 